example, in view of the current controversy I tried to find what the Encyclopedia had to say about Darwinism. I was surprised to find that it was an entry neither in the main text nor in the index. There are, it is true, 11 references in the index under Darwin, Charles. None of these proved helpful, however, and the reference to the Origin of Species turned out to be no more than a brief mention in an article on plant taxonomy. After an inordinate amount of time I found what I was looking for in the article on speciation. But, incidentally, the set is silent on cladistics - at least I think it is, because it does not appear in the index.

Alan Isaacs is editor of the Macmillan Encyclopedia, the Penguin Dictionary of Science, Longman's New Dictionary of Physics, and science editor of Collins English Dictionary. He has contributed to many other dictionaries and encyclopaedias.

\section{Tests of gravity}

\author{
Joseph H. Taylor
}

Theory and Experiment in Gravitational Physics. By Clifford M. Will. ISBN 0-521-23237-6. (Cambridge University Press: 1982.) $£ 37.50, \$ 75$.

GRAVITATION, the only one of nature's basic forces that we ordinarily feel, has been the centre of renewed research effort in the past quarter. of a century - largely because improved experimental techniques have made possible ever more precise tests of theory. In a concise and meaty book, Clifford Will has summarized the recent developments in the field, and has brought together a store of information not otherwise available in one place. It is a truism that the fate of a physical theory lies in its successful prediction of the results of experiments. With this fact clearly in mind, Professor Will presents his material by concentrating on the methods and results of both laboratory and astronomical tests of the subtleties of gravity, and on the theoretical tools used for interpreting the experiments.

The first quarter of the book presents the theoretical foundations of the subject, from the Einstein Equivalence Principle (in a sufficiently small, freely falling enclosure, it is impossible to tell whether gravitational forces are present) to the detailed mathematical framework of gravitation as geometry. We then encounter the Parametrized post-Newtonian (PPN) formalism, a theory of gravitational theories developed by Kenneth Nordvedt towards the end of the 1960s and later extended in collaboration with Professor Will. The PPN framework is well suited to the analysis of the slow-motion, weak-field experiments that can be performed in the laboratory or with Solar System astronomical objects; in such a post-Newtonian limit, any metric theory of gravity can be characterized by the values of a set of ten parameters, the so-called PPN parameters. For general relativity, two of the parameters have value unity and the rest are zero; in the post-Newtonian limits of other theories, the parameters have other values. The book presents detailed recipes and examples of how the parameters can be calculated within a given theory.

The middle section of the book develops the equation of motion for bodies moving under post-Newtonian conditions, and then uses this formalism to analyse the "classical" tests of general relativity: the deflection of starlight near the limb of the Sun, the time-delay of radar signals in the vicinity of the Sun and the perihelion shift of the orbit of Mercury. Together with the results of some less famous experiments involving lunar laser ranging, geophysical effects, the orbits of planets and the constancy of the Newtonian $G$, these tests establish a set of experimental constraints on the PPN parameters. We learn that "viable theory space" is squeezed to the extent that in the post-Newtonian limit, any gravitation theory must agree with general relativity to within fractional margins of error ranging from 1 to $10^{-7}$ per cent.

Unfortunately, perhaps, there are a number of distinct theories of gravity which can, by suitable adjustments of arbitrary parameters, accommodate all of the Solar System experiments. Consequently, the last quarter of the book deals with tests which probe beyond the weakfield, post-Newtonian limits. These tests involve gravitational radiation, the structure and motion of neutron stars and other inherently relativistic compact objects, timing measurements of the orbiting pulsar discovered in 1974 and, finally, observations of cosmological significance. Only the binary pulsar experiment has yet produced results of quantitative significance; again, the results are consistent with general relativity, while constraining other theories rather severely.

Why so much ado about "other" possibly viable theories of gravity, when relativity remains uncontradicted by experiment and stands alone in its freedom from arbitrarily adjustable constants? True, the failure of all attempts to quantize relativity still segregates it from the rest of modern physics, but then the other theories being discussed are not quantum theories either. So the motivation for undertaking the hard labours involved in developing or mastering the PPN machinery seems obscure. Nevertheless, I find Professor Will's book a valuable addition to my library, and a most useful reference work. Other researchers and serious students of gravitation should be pleased with it, too.

J.H. Taylor is Eugene Higgins Professor of Physics at Princeton University.

\section{Seen in colour}

\author{
W. R. A. Muntz
}

Comparative Color Vision. By Gerald $\mathrm{H}$. Jacobs. Pp.209. ISBN 0-12-378520-0. (Academic: 1982.) £16, \$24.

THE opening words of the preface to Comparative Color Vision describe how the author received a telephone call some years ago asking how to go about studying colour vision in the American elk. Anyone who has worked on comparative colour vision will have received similar requests, and will have shared Dr Jacobs's frustration that no convenient and up-to-date source of information is available on the methods and results of work in this area.

The present book is Dr Jacobs's response to this situation, and about half of it does indeed give the required information both clearly and concisely. Thus an initial chapter sets out the necessary techniques, dealing particularly with the bugbear problem of brightness, and should help anyone designing experiments to avoid the major pitfalls. Two further chapters late in the book present a comparative survey of colour vision in vertebrates (invertebrates are specifically excluded). These chapters are especially comprehensive in their coverage of the mammals, particularly the primates: 36 of the 58 pages of the survey deal with the mammals, and of these 20 are devoted to the primates. This emphasis is not a defect, for it is only reasonable for a book to reflect the author's interests, but it inevitably results in the "lower" vertebrates being more sketchily covered. With this minor proviso, these chapters provide a valuable introduction to the field.

The rest of the book is mostly devoted to background information on vision in general, with accounts of the structure of the eye, photopigments, basic electrophysiology, the psychophysics of human colour vision and so on. I feel these sections are less useful, since many other publications exist which deal with these subjects well. It is also impossible, in a book of this length, to cover such a range of topics in any depth, and it seems to me that in this part of the book the author has fallen between two stools: the information is so condensed that it would be difficult for anyone who is not already familiar with the field to understand it, and yet for those who are experienced it will add little to their knowledge or understanding.

In spite of these reservations the book serves a useful purpose. Anyone working within the general area it covers will find both interest and a number of new facts, and it should in particular provide a very useful introduction for someone who knows the basic data of vision, and now wishes to pursue the subject in colour.

W.R.A. Muntz is Professor of Biology at the University of Stirling. 